\title{
Indigo (Cat5 \& Sep5) System Mechanical Thrombectomy For Acute Mesenteric Ischemia: An Innovative Approach for Treatment of a Severe but Not So Infrequent Disease
}

\author{
De Gregorio MA*, Guirola JA, Mayoral and Sierre S \\ Minimally Invasive Techniques Research Group GITMI, Spain
}

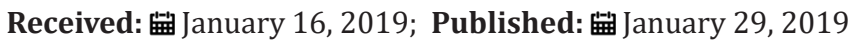

*Corresponding author: MA de Gregorio, Minimally Invasive Techniques Research Group GITMI, Spain

Keywords: Mechanical Thrombolysis; Throboaspiration; Superior Mesenteric Artery; Acute Mesenteric Ischemia

Abbreviations: AMI: Acute Mesenteric Ischemia; MVT: Mesenteric Venous Thrombosis; NOMI: Non-Occlusive Mesenteric Ischemia; CTA: Computerized Tomography Angiography; SMA: Superior Mesenteric Artery; CDT: Catheter Directed Thrombolysis; OSG: Compared Open Surgery Group

\section{Introduction}

Acute Mesenteric ischemia (AMI) is a severe and commonly underreported disease that has an annual incidence of 0.09 $0.2 \%$ per patient year [1]. However some studies have shown an increased incidence of AMI after performing an exhaustive analysis of 402 autopsies and surgeries (1970-1982 Malmo, Sweden) [2]. All mesenteric ischemia (MI) has and incidence of 12.9 cases of MI/10000 inhabitants/year, 67\% thromboembolic SMA occlusion, $16 \%$ mesenteric venous thrombosis (MVT), 15\% non-occlusive mesenteric ischemia (NOMI) and $2 \%$ have indeterminate cause [3]. We present a patient with SMA treated satisfactorily with a new thromboaspiration system.

\section{Case Presentation}

We present an 89-year-old male patient who was admitted to the emergency room with severe abdominal pain associated with diarrhea. Upon admission the ECG showed an atrial fibrillation and high lactate levels associated with leukocytosis (Figures 1A \& 1B). An abdominal computerized tomography angiography (CTA) (Figure 2A) revealed a complete occlusion of the superior mesenteric artery (SMA) with a hypodense image that suggested an intravascular thrombus; however, there were no pathological findings in the small or large Bowel. The interventional radiologist team was called to perform a diagnostic angiography and thrombolysis.

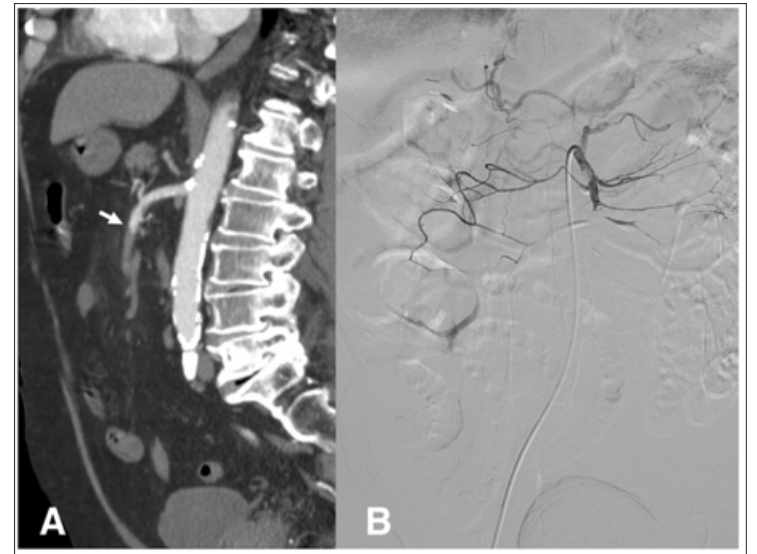

Figure 1:

a. Sagittal view CT angiography, white arrow shows complete occlusion of the superior mesenteric artery (SMA) with a hypodense image suggesting thrombus.

b. Diagnostic angiography shows a thrombosis of the SMA.

The angiography revealed a complete occlusion of the SMA near the origin, a 6 Fr guide catheter $(55 \mathrm{~cm}$ length, Flexor Ansel Guiding sheath Cook Medical Bloomington USA) was introduced 
into the SMA and the Indigo CAT5 aspirator tubing and separator (PENUMBRA Indigo system, Alameda California USA) was used for thromboaspiration, and also catheter directed thrombolysis (CDT) with 500,000.00 UI of urokinase was administered. After 10-15 minutes a complete extraction of the thrombus was performed with some residual occlusion in jejunal and ileal arteries as a result of distal embolization of small thrombi (Figure 2B). A 5 Fr multiperforated catheter $(100 \mathrm{~cm}$ length and $10 \mathrm{~cm}$ infusion length; Cragg-Mcnamara Cath ev3 Ltd. Paris France) was introduced all the way until the ileocolic artery and thrombolysis perfusion was initiated with 50,000.00 UI/ of urokinase every hour in a total of 24 hours (Figure 2C).

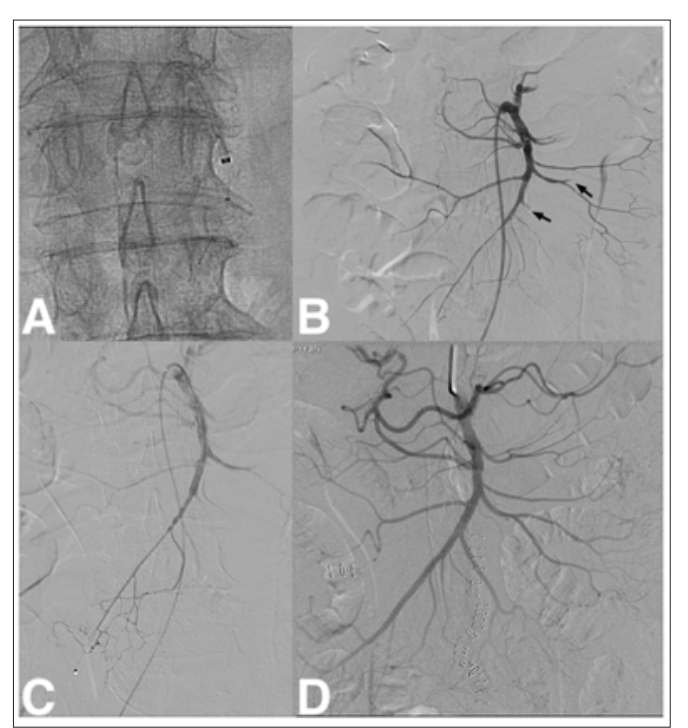

\section{Figure 2:}

a. CAT5 and SEP5 indigo system by Penumbra.

b. Final result after thromboaspiration with CAT5 Indigo System, black arrows shows residual and distal thrombosis of yeyunal and ileal artery branches.

c. Multiperforated infusion catheter for Urokinase (UK) perfusion (50,000.00 UI UK/Hr.)

d. Angiography control after $24 \mathrm{hrs}$ of UK perfusion, notice the metallic surgical stapling as a result of laparoscopic procedure done by the surgical to discard intestinal complications.

The surgery team did an exploratory laparoscopy after the procedure to determine the viability of the intestines, which showed no pathological findings. After 24 hrs a control SMA angiography was performed with a complete revascularization (Figure 2D), and without any residual thrombi in jejunal or ileal artery branches. The surgery team carried out a second look laparoscopy, to discard any abdominal complications. The cardiology department suggested acenocumarol treatment as anticoagulant therapy for the cardiac arrhythmia, and the patient was discharged after 5 days after admission.

\section{Discussion}

The time elapsed from the onset of symptoms to the diagnosis of AMI is substantial, although clinical symptoms may be imprecise especially in elderly patients [4]. Currently the early diagnosis of AMI is possible thanks to the multiple phases CT-angiography that presents high sensibility and specificity. CTA can establish the diagnosis of AMI but also rule out other pathologies with similar symptoms [1]. Some authors conclude that the signs, symptoms and laboratory tests are insufficient for the diagnosis of AMI and the CTA has a better diagnostic accuracy [4]. In our patient the clinical presentation had the typical abdominal pain, high lactic and D-dimer levels. However, CTA was able to diagnose the thromboembolic event with a rapid response and great precision. The mortality due to acute mesenteric ischemia has not changed in the last years despite the improvement of the treatments especially endovascular procedures. The overall rate of AMI mortality is very high, with a $38 \%$ of early mortality [4] of the total number of patients diagnosed.

The study performed by Eslami et al. included a total of 42,154 patients (U.S. study 2003-2011), in which they compared open surgery group (OSG) versus endovascular group (EVG); the study reported significantly higher mortality rates and small bowel resections for the OSG (21,9\% versus $15,3 \%$ and $14,9 \%$ versus 9\%, respectively) [5]. Currently, a hybrid treatment (open and endovascular surgery) is the treatment of choice in patients with AMI. Having ruled out peritonitis requiring laparotomy, revascularization of the mesenteric artery in open or endovascular surgery is recommended prior to surgery of the intestines [5]. Some studies have performed an endovascular revascularization of a thrombosed SMA using a 6 Fr catheter with aspiration followed or not by local fibrinolysis through a multiperforated catheter $[6,7]$.

In our case we used the Indigo CAT5 Fr thrombectomy system with SEP5 (Indigo System, by PENUMBRA) that combines thrombus fragmentation and aspiration with very satisfactory immediate result. In our knowledge it is the first published case of recanalization of AMI occluded by thrombosis. If there are any doubts regarding the intestinal integrity or any other abdominal organs, laparotomy o laparoscopic surgery must be performed. During the procedure, intestinal colour changes and transit disturbances have to be monitored, in order to know if a surgical intervention is needed. In our patient, laparoscopic surgery was performed, which showed no intestinal damage. The use of thrombectomy systems (fragmentation and aspiration) connected to a suction pump may improve the use of single catheters [8] (not specifically designed for this purpose). Although some authors have used endovascular aspiration catheters to treat AMI [7]. In our knowledge it is the first reported case that describes the uses of an Indigo type catheter connected to vacuum for this use. The Indigo system is a simple but effective device for thromboaspiration, as well as a CDT. The only inconvenient is the price compared with other conventional catheters. It is necessary to acquire more experience and to carry out comparative studies to establish efficiency and cost analysis between devices.

\section{Ethical Approval}

All procedures performed in studies involving human participants were in accordance with the ethical standards of the 
institutional and/or national research committee and with the 1964 Helsinki declaration and its later amendments or comparable ethical standards.

\section{Informed Consent}

Informed consent was obtained from all individual participants included in the study.

\section{References}

1. Cudnik MT, Darbha S, Jones J, Macedo J, Stockton SW, et al. (2013) The diagnosis of acute mesenteric ischemia: A systematic review and metaanalysis. Acad Emerg Med 20(11): 1087-100.

2. Acosta S, Ogren M, Sternby NH, Bergqvist D, Björck M (2004) Incidence of acute thrombo-embolic occlusion of the superior mesenteric artery--a population-based study. J Eur Soc Vasc Surg 27(2): 145-150.

3. Acosta S (2015) Mesenteric ischemia. Curr Opin Crit Care 21(2): 171178.

\section{ISSN: 2574-1241}

DOI: $10.26717 / B J S T R .2019 .13 .002451$

MA de Gregorio. Biomed J Sci \& Tech Res

(C) (P) This work is licensed under Creative

Submission Link: https://biomedres.us/submit-manuscript.php
4. Kärkkäinen JM, Lehtimäki TT, Manninen H, Paajanen H (2015) Acute Mesenteric Ischemia Is a More Common Cause than Expected of Acute Abdomen in the Elderly. J Soc Surg Aliment Tract 19(8): 1407-14.

5. Eslami MH, Rybin D, Doros G, McPhee JT, Farber A (2016) Mortality of acute mesenteric ischemia remains unchanged despite significant increase in utilization of endovascular techniques. Vascular 24(1): 4452.

6. Heiss P, Loewenhardt B, Manke C, Hellinger A, Dietl KH, et al. (2010) Primary percutaneous aspiration and thrombolysis for the treatment of acute embolic superior mesenteric artery occlusion. Eur Radiol 20(12): 2948-2958.

7. Acosta S, Björck M (2014) Modern treatment of acute mesenteric ischaemia. Br J Surg 101(1): e100-108.

8. Yamada R, Adams J, Guimaraes M, Schönholz C (2015) Advantages to Indigo mechanical thrombectomy for ALI: device and technique. J Cardiovasc Surg (Torino) 56(3): 393-400.

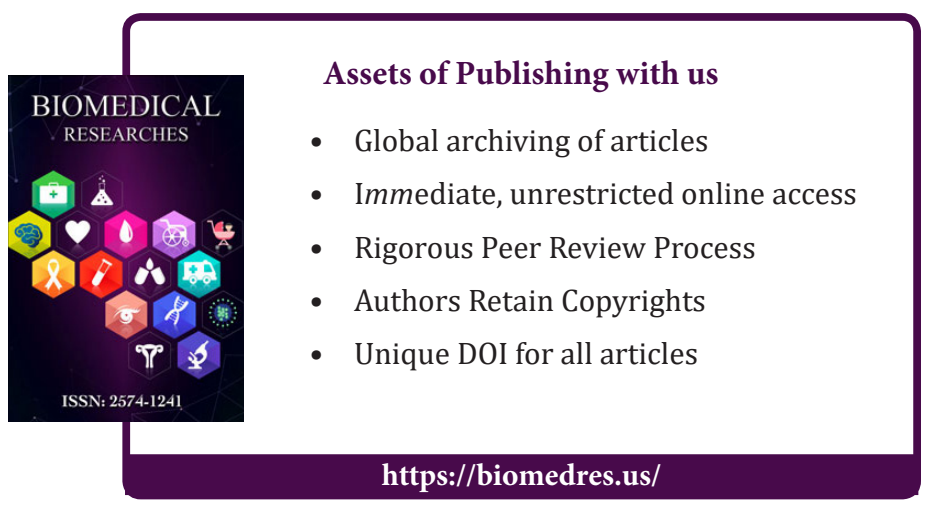

